\title{
The Impact of Provider Websites on Patient Decision Making
}

\author{
Rita A. DiLeo \\ University of Scranton
}

\author{
William F. Miller \\ University of Scranton
}

Marissa F. Lembo

University of Scranton

\author{
Alexandra L. Coviello \\ University of Scranton
}

It is imperative for health care providers to demonstrate performance excellence through a virtual exposure. Currently, providers use various online strategies to capture consumers' attention, and by analyzing existing approaches, provider specific strategies will be identified that will enhance organizational virtual planning and increase market share. Achieving superiority by leveraging competitive advantage, highlighting recognitions, and eliminating wasteful information is vital from an operational and marketing perspective. By addressing patient questions and concerns through simplified website navigation, providers could 'tip' patient decision making in their favor. This research explores the importance and impact of the patient online experience and reveals current flaws on provider webpages.

Keywords: performance excellence, online strategies, provider websites, marketing, competitive advantage, patient decision making

\section{INTRODUCTION}

As technology continues to provide healthcare professionals with breakthroughs in research, medicine, and innovative therapies, it has a substantial impact on patient decision-making. Using technology to build a strong online presence can assist healthcare organizations' efforts to market innovative services, better reflect their current position in the market, and facilitate a patient-centric approach to care. Simply stated, a provider website, may influence market share, affect patient experience, and impact quality of care (Sorondo, et al., 2016).

In order to build this strong online presence, it is crucial for health care organizations to complete periodic assessment and recognize where they stand in the virtual world. A comparative analysis of online platforms in academic medical centers was completed to better understand aspects driving consumer usage 
of these large, complex health systems. The indicators which were analyzed relate to quality of information, ease of navigation, access to patient portals, and drivers of patient satisfaction.

\section{FINDINGS}

The first health system whose online presence was reviewed is Johns Hopkins Medicine (JHM) located in Baltimore, Maryland. JHM includes The Johns Hopkins Hospital, Johns Hopkins Bayview Medical Center, and Johns Hopkins Community Physicians. As one would assume given the current pandemic, COVID-19 is the predominant subject on the JHM website. However, conspicuously absent on the main page is any information relating to rankings, awards, and accreditations.

The JMH site is easily navigated with multiple links covering subjects such as patient safety, primary and specialty care, surgery procedures, and hospital and emergency care. They also include an "Explore Johns Hopkins Medicine" banner for further exploration. Choosing the "Health" link leads one to an informational page on COVID-19 and other community health and wellness items while other links explore patient care, research, the medical school, and overall organization information.

Known for its cutting edge research, JHM provides access to information on "The innovative research conducted at Johns Hopkins Medicine [that] is vital to both medical advances and unsurpassed patient care." (Johns Hopkins Medicine, 2021), which is happening within the organization, at the click of a button. Consumers could navigate through extensive research topics including cancer, aging, heart, neurology, Coronavirus, diabetes, Cochlear Implants, gastroenterology and hepatology, reproductive sciences and women's health, dermatology, radiology, genetics, and genome biology.

If the consumer chooses to investigate more within these sections, they are conveniently brought to an additional page where they are provided with substantial information on each active study. Further, within this page, there is a link titled "Help Figuring Out What I Need." Consumers are brought to The Institute for Clinical and Translational Research (ICTR) Navigator which helps to direct them and investigators on their next steps in clinical research within the organization. An area to submit an ICTR Connection Request provides consumers with an option to communicate with professionals on a specific topic.

Further review found the organization's website provides a well-rounded overview of the education and training within the hospital. This feature supplies prospective applicants with a virtual tour of The Johns Hopkins Hospital and Johns Hopkins Bayview Medical Center and their education offerings. Potential students could learn more about life as a medical student, graduate student, resident, postdoc or fellow. In addition, the award-winning blog titled "Biomedical Odyssey" is available to the general public on their website. This blog helps future scholars to become acquainted with life as a student in the health professions, studying at JHM. Although it targets new students, it offers a wide range of demographics the chance to better understand medical education and the health professions.

JHM developed an accessible and straight forward way to identify specialties and specialists, as well as connect with physicians in any of their three locations. The search bar provides patients the ability to enter physician last name, specialties, or keywords. Using this search, potential patients can find physicians and established patients can research out to their provider.

The use of short videos and podcasts throughout the site gives patients visual and audio representations that enhance written information, instructions, and services. For example, JHM has established a "COVID19 Resource Center" with a self-checker, vaccine information, telemedicine services, general information, visitor guidelines and expanded patient care options. The information covers a broad variety of services to reduce common misconceptions and transmission rates, and can be utilized by any consumer, free of charge, to check symptoms, protect themselves and others, and access parent and caregiver information.

Patients can open their patient portal and access MyChart for test results and appointment information or access a "Find Your Way" App for assistance with a wide variety of information and tasks. These features include the following: navigating the buildings of The Johns Hopkins Hospital and Johns Hopkins Bayview Medical Center, finding a doctor, and making an appointment, reviewing health information and access to the digital library. Key landmarks are noted within the system with step-by-step directions to appointments, locating parking spots, amenities, and service. 
The next organization reviewed was the Mayo Clinic. With locations in Minnesota, Arizona, Florida, London, and Abu Dhabi, The Mayo Clinic is a non-profit, academic medical center which provides distinguished, integrated care to local, national, and international patients (mayoclinic.org, 2021). Strategically located on the website is a link noting the organization's multiple top rankings and links to other organizational awards and accreditations and links emphasizing the organization's international services and the ability to donate to Mayo Clinic.

At the top of the website, one can find single click access to COVID-19 information, vaccine updates, and care guides. Using the COVID-19 page within the website, individuals can see overall recommendations, recent trends, visitor policies and guidelines for each of the Mayo Clinic locations, and additional pandemic related resources for medical professionals, employers, and researchers. They even have a process by which individuals can donate to COVID-19 research.

The rest of the website contains links provides consumers with easy access to most everything one could ask for in a website. The information is informative and organized into various categories which include Diseases and Conditions A-Z, Tests and Procedures A-Z, Drugs and Supplements A-Z, Symptoms A-Z, Research and Health Letters, Healthy Living Program, Health Books, and a "For Medical Professionals" Section. Each of these categories provides in-depth medical knowledge for patients and providers.

The Mayo Clinic's patient portal App under their "Patient Online Services" can be utilized for appointment itineraries, maps and resources, appointment requests, secure messaging online telehealth care, lab results, and radiology imaging results. This App is also useful for providing practical advice from physicians, dieticians and fitness experts. This service is accessible to all patients who create an App account and are under the care of a physician associated with the Clinic. It is an easy, rapid and reliable way to correspond with a trained medical professional.

On the main page, the section titled "International Patients" provides information to those around the globe looking to seek out their exceptional services. Patients, no matter their location, are able to quickly and easily request an appointment and view the financial services of the organization. The subsection titled "Language Services" shows just how crucial it is for the Mayo Clinic to establish and maintain a culturally competent environment.

The Concierge Services section of the site aims to provide current and prospective patients with services and appointments to make the visit as comfortable and convenient as possible. For example, this section promotes the service by advertising the benefits. The page helps patients book travel arrangements, hotels, notarize documents, and obtain directions. It is a service devoted to ensuring the patient and their family focus on what is most important; their medical care. The website offers "Advice From Other Patients" section allowing future patients to connect with those who are or already have received care at the organization. This unique blog format encourages patients to ask questions, share their stories, and offer support to other patients.

Also prominently found on the website is a link to the College of Medicine and Science. Here one can find information on not only the medical school but also graduate programs in Biomedical Sciences, the Mayo Clinic School of Health Sciences, and their programs in graduate medical education and continuous professional development. Individuals at all levels of the health education spectrum can find programs that fit their needs.

The third academic medical center website reviewed is Houston Methodist (HM) with several locations in the Greater Houston, TX area. Houston Methodist Hospital is the premier hospital in the system and joins six community hospitals and Texas Medical Center to form the HM system. The system website has a wide set of resources for patients and healthcare professionals. In addition to the primary navigation links ("For Patients", "Services \& Specialties", "For Health Professionals", "Research", "Education", and "Locations"), the home page contains a large link that navigates users to the organization's various rankings, awards, and accreditations.

An extremely convenient resource that HM provides on their website is their Coronavirus Resource Center. As their home page succinctly states when hovering over the Coronavirus Resource Center link, one can find "The Latest Information, Resources and Answers You Need" (Houston Methodist, 2021). It 
provides patient and visitor information, answers about COVID-19, access to virtual care, a free symptom checker, and doctor/hospital appointments. It also provides a list of concerns that connect the user with care options and resources.

Contained in the patient tab is the scheduling system with a unique design dividing doctors' appointments, imaging, and virtual urgent care information. The patient tab also provides insurance information allowing patients to learn if their insurance is accepted at whichever HM location they would be receiving treatment, as well as easy access to their patient portal. Through HM's patient portal, referred to as MyChart, the health care experience of patients has been enriched. Patients are able to initiate easy communication with providers, review and pay their medical bills online, and get price estimates for the cost of care. In addition, the portal gives patients the option to view their medications, immunization history, and test results when posted, and doctor's comments. One element of MyChart enhancing the online health care experience is the ability to schedule one's upcoming appointment and view details of past appointments.

Additional components used to increase patient satisfaction focus on "Out of Town Patients" with a section dedicated to explaining services offered, second opinions, travel accommodations, and a Houston guide. One last section driving users to provider facilities is a section titled "Why Choose Houston Methodist." It explains the organization's U.S. News National Rankings, other honors, patient stories, locations, The Patient Experience, and Quality Resources. Elements such as this on a provider website can help guide a patient make their choice when deciding where to receive care.

As with the other sites reviewed, Houston Methodist offers links to the Research and Education components within their organization. The Research dropdown is headlined by "Innovations with Impact Research that brings the latest advances to our patients" (Houston Methodist, 2021). The Education dropdown provides links for patients and potential students seeking continuing medical education, leadership fellowships, and medical, research, and nursing education.

The final healthcare organization site reviewed is University of California, Los Angeles Health (UCLA) which comprises numerous hospitals, as well as primary care facilities in the Los Angeles area. The first two resources offered through the website are a LEARN MORE link for "What you need to know about COVID-19" (UCLA Health, 2021) and an instant chat feature. The instant chat feature is stationary and does not scroll as the website does, thus it is always accessible.

The UCLA Health website offers a division of each hospital facility throughout the system to include breakdown of locations such as primary care, outpatient surgery, imaging, etc. Also offered is an interactive map for consumers. After selecting a facility, consumers are brought to another page that explains more information regarding that facility.

In addition, UCLA Health includes a Health Encyclopedia for consumers to access. Detailed topics can be accessed regarding conditions, health centers, drugs/supplements, nutrition/general wellness, family, news center, healthy living, and tools/multimedia. Patients may find this a great resource to obtain information about children, older adults' health, mental health, smoking cessation, weight management, etc.

Additional interesting resources for patients on the UCLA Health website are found on within the "Preparing for Surgery Checklist." This section provides videos for the patient depending on in which facility their surgery would be taking place. Health forms, instructions, and patient guides are also provided for patients to use to prepare. This is a helpful resource for patients and could possibly influence their comfort level prior to surgery. UCLA Health provides a video library regarding conditions and treatment varying from children's health to geriatric care. One last resource that UCLA provides is a drug interaction checker to help determine if medications have adverse interactions with another medicine or vitamin.

"myUCLAHEALTH" is the UCLA Health patient portal. It allows patients to get directions and maps to more than 180 medical practice locations, as well as has the ability to access health records for patients. Through this platform, patients are able to find out more about medical services, watch webinars, health education videos, and utilize the extensive health library. Patients are also able to request appointments and schedule directly with the doctor themselves.

As one would expect from UCLA Health, they provide a link to what is happening with their Clinical Research, which is split between All Clinical Trials and COVID-19 Clinical Research. However, All 
Clinical Trials page does not provide a listing or examples of the current research. It connects the user to a keyword or department search through which the user is able to search for specific areas of research.

The home page is longer than the others reviewed with patient stories, FIND A PROVIDER, multiple COVID-19 resources, health news, wellness, and provider profile sections. It takes some scrolling on the home page in order to find a link to the system's U.S. News and World Report rankings. Plus, except for a small link (School of Medicine) at the top of the home page, links to the medical, nursing, or dentistry schools are not obvious. They are located under a link titled For Healthcare Professionals.

\section{DISCUSSION}

Through comparative analysis of these four major academic medical health systems' online platforms based on quality of information, ease of navigation, access to patient portals, and drivers of patient satisfaction, we have established suggestions health systems should consider in order to enhance their visual presence.

1. Easy to access portals with clear steps/set of instructions for navigation

2. Information: Quality over quantity, easy to read, language interpretation services

3. Easy scheduling of services and appointments

4. Chat features

5. Concierge services for patients

6. Use of short videos and podcasts

7. Advice from other patient blogs to share experience and offer support, insight, and tips

8. COVID-19 information and self-symptom check

The above suggestions were established based on commonalities found between each of the four major health systems' websites. To manage patients' expectations during the COVID-19 pandemic, it appears healthcare organizations must rapidly grow and maintain their online presence. According to Radick (2019), understanding the need for personalized care is essential to satisfaction rates - which is why options such as the chat feature, concierge services, patient blogs, and COVID-19 symptom checks are important. They offer individualized care and consideration to current and future patients. These features embedded within the websites support more value-based care models which aim to assist the patient in managing chronic diseases, COVID-19 symptoms, and easy to interpret instructions for living a healthy life, leading to more positive patient outcomes. The services embedded within the site offer health consistency throughout the patient's life, rather than solely treating them when they are sick. These services are available as preventative measures.

Each of the suggestions above also support a more convenient approach to seeking care in an atypical setting. The suggestions including the easy access to the portal with a clear set of steps and instructions, the manageable scheduling of services, the chat features, concierge services, short videos and podcasts, patient blogs and COVID-19 self-symptom check also manage a dialogue between the patient and provider which can assist in providing the patient with a more enhanced standard of care. (Radick, 2019)

Radick reports age also has an effect on choice of provider. He notes these differences could be attributed to provider digital capabilities and ease of access to test results.

Younger consumers are much more likely to choose medical providers with digital capabilities, according to the 2019 Accenture digital health consumer survey. For example, 40 percent of Generation $\mathrm{Z}$ (born since 1997) and 44 percent of millennials (1981-1996) indicate that easy access to test results via mobile or online channels would increase the likelihood of choosing a medical provider offering such capabilities. (p. 10)

Having access to these features easily available for younger generations will mean a greater chance of retaining Generation $\mathrm{Z}$ and millennial patients. However, Radick also notes a growing interest in ease of access to results in Generation $X$, so it is imperative for providers to offer these services.

Taneja (2020) explains the critical need for pivoting areas in healthcare that were once in-person to online platforms. Many organizations who have added these advanced search options, instructions and services to their online websites have assisted in flattening the COVID-19 curve. With more resources 
available at the click of a button from a trusted, world renowned organization, more patients will be able to treat their conditions from their home while corresponding with a trained medical professional through the chat feature or patient portal area of the site, as well as monitoring their symptoms through the "selfsymptom check". Their viewing of step by step, transparent instructions on the websites podcast or short video link could be critical in reducing viral transmission and saving lives. Accessing website patient blogs could establish a support system with other patients within the organization. After careful consideration and examination, implementing these suggestions would create accessible services and care for a greater population.

Virtual presence is essential in order to manage patient-centered care. Convenience and patient experience are highlighted by virtual and innovative ways to connect with the consumer (Sorondo, et al., 2016). Identifying common strategies established by four major United States health systems could allow other organizations around the United States to modify, advance, or transform their quality of information and patient portals. Establishing online services supports marketing efforts, appears to support value-based efforts and of importance, could improve patient outcomes and experiences.

All four organization sites reviewed offer advanced search options for patients to access physician contacts, step-by-step instructions on navigating patient portals to check test results, the ability to communicate with healthcare providers, and access hospital directions. They provided scheduling of appointments with the few clicks of a button and COVID-19 updates which are transparent and informative. The quality of information presented on all four of these organizations' webpages can be viewed as advantageous to consumers who are seeking out a reliable source for their healthcare needs.

Given the highly competitive environment of the healthcare industry, organizations must compete for market share against a large number of similar organizations. Organizations succeeding in capturing market share understand the target consumer population and develop effective strategies to establish a position in the market (Berkowitz, 2018). Online presence could be the first view a consumer gets of an organization and may be a driving factor in establishing market position. Website design and access to information may attract specific populations while rankings, awards, and accreditations may attract others. It is important for organizations to adapt as needed.

This need to adapt is exemplified by differences in the organizations examined. Although this research focused on healthcare organizations designated as academic medical centers or systems, there are inherent differences among them based on current local, state, and federal circumstances and organizational strengths, demographics served, geographical variations, size, and scope. As such, these differences could dictate the design, and data on websites or information deemed important could vary.

A major limitation in this study is the lack of prior studies evaluating the impact of these specific websites in this format. Hence, comparative analyses could not be completed. Furthermore, the relationship between patient satisfaction and website is challenging to assess given the lack of literature available on this topic. Additional limiting factors include:

(1) Sample size limited to four academic medical centers/systems

(2) Demographic data was not reviewed based on locations of healthcare organizations potentially influencing website design

(3) Evaluation of only the main organization's webpage was completed

(4) Patient portals could not be logged into to complete a one to one assessment across the organizations researched.

Lack of empirical evidence and scholarly research does limit comparisons; however, what was found in this review provides valuable information for the design of future studies. Since research evaluating these websites is absent, opportunities to assess the overall influence of websites on patient satisfaction via focus groups or surveys exists. Future studies comparing website usage within a specific academic medical system across locations could potentially uncover usage patterns based on patient demographics. Further, a detailed analysis of patient comments could be helpful in determining a variety of influences on patient satisfaction

While all websites explored offer education links, it is unknown if a lack of culturally relevant patient education exists, thus offering another opportunity for research. Identifying types of information most 
needed by the specific patient populations that protects their confidentiality and enhances health could be explored. Moreover, research is needed to evaluate the content available on medical providers within the system such as their clinical research interests or personal biography. Finally, engaging one of the academic medical systems in this type of research could be of value.

Another constraint of this study is that it is limited to academic medical centers. These organizations tend to have greater resources at their disposal that could be used to develop their online presence. Additional research could be developed that examines the presence of specific website features as a result of available resources. This new research could also be expanded to include comparing patient satisfaction rates stratified by organizational revenue or other demographic variables.

Another point that is important to remember is this review did not include non-provider website based channels. A growing number of providers, and insurers alike, are moving into mobile based digital channels. The ease of use of a smartphone or tablet that allows for portable access to test results and appointment generation or follow-up is an important element of an organization's virtual footprint. Additional research could be completed that includes a review of these components.

\section{REFERENCES}

Berkowitz, E.N. (2018). Essentials of health care marketing (4th ed.). Sudbury, MA: Jones \& Bartlett. Houston Methodist. (2021, January 15). The Difference Between Practicing Medicine and Leading It. Retrieved from https://www.houstonmethodist.org/

Johns Hopkins Medicine. (2021, January 15). Retrieved from https://www.hopkinsmedicine.org/research/index.html

Mayo Clinic. (2021, January 15). Retrieved from www.mayoclinic.org/

Radick, L.E. (2019). Consumer engagement beyond the app. Healthcare Executive, 34(5), 9-16.

Sorondo, B., Allen A., Fathima, S., Bayleran, J., \& Sabbagh, I. (2017). Patient portal as a tool for enhancing patient experience and improving quality of care in primary care practices. The Journal of Electronic Health Data and Methods, 4(1), 31. DOI: http://dx.doi.org/10.13063/23279214.1262

Taneja, H. (2020, April 28). How tech companies can help fix U.S. health care. Harvard Business Review. Retrieved from https://hbr.org/2020/04/how-big-tech-can-help-fix-u-s-health-care

UCLA Health. (2021, January 15). Retrieved from https://www.uclahealth.org/ 